\title{
Backward stochastic differential equations and Feynman-Kac formula for Lévy processes, with applications in finance
}

\author{
DAVID NUALART ${ }^{1}$ and WIM SCHOUTENS ${ }^{2}$ \\ ${ }^{1}$ Universitat de Barcelona, Gran Via de les Corts Catalanes 585, E-08007 Barcelona, Spain. \\ E-mail: nualart@mat.ub.es \\ ${ }^{2}$ Katholieke Universiteit Leuven, Celestijnenlaan 200B, B-3001 Leuven, Belgium. \\ E-mail: Wim.Schoutens@wis.kuleuven.ac.be
}

In this paper we show the existence and uniqueness of a solution for backward stochastic differential equations driven by a Lévy process with moments of all orders. The results are important from a pure mathematical point of view as well as in the world of finance: an application to Clark-Ocone and Feynman-Kac formulas for Lévy processes is presented. Moreover, the Feynman-Kac formula and the related partial differential integral equation provide an analogue of the famous Black-Scholes partial differential equation and thus can be used for the purpose of option pricing in a Lévy market.

Keywords: backward stochastic differential equations; Lévy processes; option pricing; orthogonal polynomials

\section{Introduction}

In the first paper concerned with backward stochastic differential equations (BSDEs), Bismut (1973) introduced a nonlinear Ricatti BSDE and showed the existence and uniqueness of bounded solutions. Pardoux and Peng (1990) considered general BSDEs, and this paper was the starting point for the development of the study of these equations (see Pardoux 1998). Moreover, interest in these equations is not confined to pure mathematicians - they have important applications in the theory of mathematical finance; in particular, they play a major role in hedging and nonlinear pricing theory for imperfect markets (see El Karoui and Quenez 1997; Ma and Yong 1999).

One can consider a BSDE driven by a Brownian motion as a nonlinear generalization of the integral representation theorem for square-integrable martingales. Then it is natural to extend such equations to the case of Lévy processes, that is, processes with independent and stationary increments. We recall that a Lévy process consists of three stochastically independent parts: a purely deterministic linear part, a Brownian motion and a pure jump process. Situ (1997) studies BSDEs driven by a Brownian motion and a Poisson point process. Ouknine (1998) considers BSDEs driven by a Poisson random measure. In both papers the main ingredient is the integral representation of square-integrable random variables in terms of a Poisson random measure (see Jacod 1979). 
Nualart and Schoutens (2000) proved a martingale representation theorem for Lévy processes satisfying some exponential moment condition. The purpose of the present paper is to use this martingale representation result to establish the existence and uniqueness of solutions for BSDEs driven by a Lévy process of the kind considered in Nualart and Schoutens (2000). The results are important from a pure mathematical point of view as well as in the world of finance. This is illustrated in the applications. The resulting Clark-Ocone and Feynman-Kac formulae are fundamental ingredients in the construction of a Malliavin calculus for Lévy process. Moreover, the Feynman-Kac formula and the related partial differential integral equation (PDIE) also have an important application in finance: they provide an analogue of the famous Black-Scholes partial differential equation (Black and Scholes 1973) and thus can be used for the purpose of option pricing in a Lévy market.

The paper is organized as follows. Section 2 contains some preliminaries on Lévy processes. Section 3 contains the main result on BSDEs driven by Lévy processes. In Section 4 we have included some applications of BSDEs driven by Lévy processes to the Clark-Ocone and Feynman-Kac formulae, and to option pricing in a Lévy market. Detailed proofs of the main results are given in the Appendix.

\section{Preliminaries}

Let $X=\left\{X_{t}, t \geqslant 0\right\}$ be a Lévy process defined on a complete probability space $(\Omega, \mathscr{F}, P)$. That is, $X$ is a real-valued process starting from 0 with stationary and independent increments and with cadlag trajectories. It is known that $X_{t}$ has a characteristic function of the form

$$
\mathrm{E}\left(\mathrm{e}^{\mathrm{i} \theta X_{t}}\right)=\exp \left[\mathrm{i} a \theta t-\frac{1}{2} \sigma^{2} \theta^{2} t+t \int_{\mathbb{R}}\left(\mathrm{e}^{\mathrm{i} \theta x}-1-\mathrm{i} \theta x \mathbf{1}_{\{|x|<1\}}\right) \nu(\mathrm{d} x)\right],
$$

where $a \in \mathbb{R}, \sigma>0$, and $v$ is a measure on $\mathbb{R}$ with $\int \mathbb{R}\left(1 \wedge x^{2}\right) v(\mathrm{~d} x)<\infty$. We will assume that, for some $\lambda>0$, the Lévy measure $v$ satisfies

$$
\int_{(-\varepsilon, \varepsilon)^{c}} \mathrm{e}^{\lambda|x|} v(\mathrm{~d} x)<\infty
$$

for every $\varepsilon>0$. This implies that the random variables $X_{t}$ have moments of all orders. Moreover, it will ensure the existence of the predictable representation (see below), which we will use in our proofs. We refer to Sato (2000) or Bertoin (1996) for a detailed account of Lévy processes.

For $t \geqslant 0$, let $\mathscr{F}_{t}$ denote the $\sigma$-algebra generated by the family of random variables $\left\{X_{s}, 0 \leqslant s \leqslant t\right\}$ augmented with the $P$-null sets of $\mathscr{F}$. Fix a time interval $[0, T]$ and set $L_{T}^{2}=L^{2}\left(\Omega, \mathscr{F}_{\mathrm{T}}, P\right)$. We will denote by $\mathscr{P}$ the predictable sub- $\sigma$-field of $\mathscr{F}_{T} \otimes \mathscr{B}_{[0, \mathrm{~T}]}$. We introduce some notation. Let $H_{T}^{2}$ denote the space of square-integrable and $\mathscr{F}_{t^{-}}$progressively measurable processes $\phi=\left\{\phi_{t}, t \in[0, T]\right\}$ such that

$$
\|\phi\|^{2}=\mathrm{E}\left[\int_{0}^{T}\left|\phi_{t}\right|^{2} \mathrm{~d} t\right]<\infty .
$$


$M_{T}^{2}$ will denote the subspace of $H_{T}^{2}$ formed by predictable processes. $H_{T}^{2}\left(l^{2}\right)$ and $M_{T}^{2}\left(l^{2}\right)$ are the corresponding spaces of $l^{2}$-valued processes equipped with the norm

$$
\|\phi\|^{2}=\mathrm{E}\left[\int_{0}^{T} \sum_{i=1}^{\infty}\left|\phi_{t}^{(i)}\right|^{2} \mathrm{~d} t\right] .
$$

Finally, set $\mathscr{H}_{T}^{2}=H_{T}^{2} \times M_{T}^{2}\left(l^{2}\right)$.

Following Nualart and Schoutens (2000), we define, for every $i=1,2, \ldots$, the so-called power-jump processes $\left\{X_{t}^{(i)}, t \geqslant 0\right\}$ and their compensated version $\left\{Y_{t}^{(i)}=\right.$ $\left.X_{t}^{(i)}-\mathrm{E}\left[X_{t}^{(i)}\right], t \geqslant 0\right\}$, also called Teugels martingales, as follows:

$$
\begin{aligned}
X_{t}^{(1)} & =X_{t}, \\
X_{t}^{(i)} & =\sum_{0<s \leqslant t}\left(\Delta X_{s}\right)^{i}, \quad i=2,3, \ldots ; \\
Y_{t}^{(i)} & =X_{t}^{(i)}-\mathrm{E}\left[X_{t}^{(i)}\right]=X_{t}^{(i)}-t \mathrm{E}\left[X_{1}^{(i)}\right], \quad i \geqslant 1 .
\end{aligned}
$$

An orthonormalization procedure can be applied to the martingales $Y^{(i)}$ in order to obtain a set of pairwise strongly orthonormal martingales $\left\{H^{(i)}\right\}_{i=1}^{\infty}$ such that each $H^{(i)}$ is a linear combination of the $Y^{(j)}, j=1, \ldots, i$ :

$$
H^{(i)}=c_{i, i} Y^{(i)}+c_{i, i-1} Y^{(i-1)}+\ldots+c_{i, 1} Y^{(1)} .
$$

It was shown in Nualart and Schoutens (2000) that the coefficients $c_{i, k}$ correspond to the orthonormalization of the polynomials $1, x, x^{2}, \ldots$ with respect to the measure $\mu(\mathrm{d} x)=$ $x^{2} v(\mathrm{~d} x)+\sigma^{2} \delta_{0}(\mathrm{~d} x):$

$$
q_{i-1}(x)=c_{i, i} x^{i-1}+c_{i, i-1} x^{i-2}+\ldots+c_{i, 1}
$$

Set

$$
\begin{aligned}
& p_{i}(x)=x q_{i-1}(x)=c_{i, i} x^{i}+c_{i, i-1} x^{i-1}+\ldots+c_{i, 1} x \\
& \tilde{p}_{i}(x)=x\left(q_{i-1}(x)-q_{i-1}(0)\right)=c_{i, i} x^{i}+c_{i, i-1} x^{i-1}+\ldots+c_{i, 2} x^{2} .
\end{aligned}
$$

Then

$$
\begin{aligned}
H_{t}^{(i)}= & \sum_{0<s \leqslant t}\left(c_{i, i}\left(\Delta X_{s}\right)^{i}+\ldots+c_{i, 2}\left(\Delta X_{s}\right)^{2}\right)+c_{i, 1} X_{t} \\
& -t \mathrm{E}\left[c_{i, i} X_{1}^{(i)}+\ldots+c_{i, 2} X_{1}^{(2)}\right]-t c_{i, 1} \mathrm{E}\left[X_{1}\right] \\
= & q_{i-1}(0) X_{t}+\sum_{0<s \leqslant t} \tilde{p}_{i}\left(\Delta X_{s}\right)-t \mathrm{E}\left[\sum_{0<s \leqslant 1} \tilde{p}_{i}\left(\Delta X_{s}\right)\right]-t q_{i-1}(0) \mathrm{E}\left[X_{1}\right] .
\end{aligned}
$$

As a consequence, $\Delta H_{t}^{(i)}=p_{i}\left(\Delta X_{t}\right)$ for each $i \geqslant 1$. In the particular case $i=1$, we obtain

$$
H_{t}^{(1)}=c_{1,1}\left(X_{t}-t \mathrm{E}\left[X_{1}\right]\right)
$$


where

$$
c_{1,1}=\left[\int_{\mathbb{R}} y^{2} v(\mathrm{~d} y)+\sigma^{2}\right]^{-1 / 2}
$$

and

$$
\mathrm{E}\left[X_{1}\right]=a+\int_{\{|z| \geqslant 1\}} z v(\mathrm{~d} z) .
$$

In the case $\int_{\mathbb{R}}|z| v(\mathrm{~d} z)<\infty$, assuming $a=\int_{\{|z|<1\}} z v(\mathrm{~d} z)$, we obtain $\mathrm{E}\left[X_{1}\right]=\int_{\mathbb{R}} z v(\mathrm{~d} z)$.

The main result in Nualart and Schoutens (2000) is the predictable representation property (PRP): every square-integrable random variable $F \in L_{T}^{2}$ has a representation of the form

$$
F=\mathrm{E}[F]+\sum_{i=1}^{\infty} \int_{0}^{T} Z_{s}^{(i)} \mathrm{d} H_{s}^{(i)}
$$

where $Z_{t}$ is a predictable process in the space $M_{T}^{2}\left(l^{2}\right)$.

Remark. If $v=0$, we are in the classical Brownian case and all non-zero degree polynomials $q_{i}(x)$ will vanish, giving $H_{t}^{(i)}=0, i=2,3, \ldots$. If $\mu$ only has mass at 1 , we are in the Poisson case; here also $H_{t}^{(i)}=0, i=2,3, \ldots$. Both cases are degenerate in this Lévy framework.

From these observations, it is not so hard to see that the PRP property shows that financial markets, with a stock price behaviour $S_{t}=\exp \left(X_{t}\right)$, based on a non-Brownian or non-Poisson Lévy process $X_{t}$, are incomplete, meaning that perfect replicating or hedging strategies do not exist for all relevant contingent claims.

\section{BSDE for Lévy processes}

Taking into account the results and notation presented in the previous section, it seems natural to consider the BSDE

$$
-\mathrm{d} Y_{t}=f\left(t, Y_{t-}, Z_{t}\right) \mathrm{d} t-\sum_{i=1}^{\infty} Z_{t}^{(i)} \mathrm{d} H_{t}^{(i)}, \quad Y_{T}=\xi,
$$

where $H_{t}^{(i)}$ is the orthonormalized Teugels martingale of order $i$ associated with the Lévy process $X ; f: \Omega \times[0, T] \times \mathbb{R} \times M_{T}^{2}\left(l^{2}\right) \rightarrow \mathbb{R}$ is a measurable function such that $f(\cdot, 0,0) \in H_{T}^{2} ; f$ is uniformly Lipschitz in the first two components, that is, there exists $C>0$ such that $\mathrm{d} t \otimes \mathrm{d} P$ almost surely, for all $\left(y_{1}, z_{1}\right)$ and $\left(y_{2}, z_{2}\right)$ in $\mathbb{R} \times l^{2}$,

$$
\left|f\left(t, y_{1}, z_{1}\right)-f\left(t, y_{2}, z_{2}\right)\right| \leqslant C\left(\left|y_{1}-y_{2}\right|+\left\|z_{1}-z_{2}\right\|_{l^{2}}\right) ;
$$

and, finally, $\xi \in L_{T}^{2}$. If $(f, \xi)$ satisfies these assumptions, the pair $(f, \xi)$ is said to be 
standard data for the BSDE. A solution of the BSDE is a pair of processes, $\left\{\left(Y_{t}, Z_{t}\right)\right.$, $0 \leqslant t \leqslant T\} \in H_{T}^{2} \times M_{T}^{2}\left(l^{2}\right)$, such that the following relation holds for all $t \in[0, T]$ :

$$
Y_{t}=\xi+\int_{t}^{T} f\left(s, Y_{s-}, Z_{s}\right) \mathrm{d} s-\sum_{i=1}^{\infty} \int_{t}^{T} Z_{s}^{(i)} \mathrm{d} H_{s}^{(i)} .
$$

Note that the progressive measurability of $\left\{\left(Y_{t}, Z_{t}\right), 0 \leqslant t \leqslant T\right\}$ implies that $\left(Y_{0}, Z_{0}\right)$ is deterministic.

A first key result concerns the existence and uniqueness of the BSDE solution.

Theorem 1. Given standard data $(f, \xi)$, there exists a unique solution $(Y, Z)$ which solves the BSDE (2).

The proof of Theorem 1, and of Theorem 2 below, can be found in the Appendix. Theorem 2 states the continuous dependency of the solution $(Y, Z)$ on the final data $\xi$ and the function $f$.

Theorem 2. Given standard data $(f, \xi)$ and $\left(f^{\prime}, \xi^{\prime}\right)$, let $(Y, Z)$ and $\left(Y^{\prime}, Z^{\prime}\right)$ be the unique adapted solutions of the BSDE (2) corresponding to $(f, \xi)$ and $\left(f^{\prime}, \xi^{\prime}\right)$. Then

$$
\begin{aligned}
& \mathrm{E}\left[\int_{0}^{T}\left(\left|Y_{s-}-Y_{s-}^{\prime}\right|^{2}+\sum_{i=1}^{\infty}\left|Z_{s}^{(i)}-Z^{\prime(i)}\right|^{2}\right) \mathrm{d} s\right] \\
\leqslant & C\left(\mathrm{E}\left[\left|\xi-\xi^{\prime}\right|^{2}\right]+\mathrm{E}\left[\int_{0}^{T} \mid f\left(s, Y_{s-}, Z_{s}\right)-f^{\prime}\left(s, Y_{s-},\left.Z_{s}\right|^{2} \mathrm{~d} s\right]\right) .\right.
\end{aligned}
$$

\section{Applications}

Suppose our Lévy process $X_{t}$ has no Brownian part, that is, $X_{t}=a t+L_{t}$, where $L_{t}$ is a pure jump process with Lévy measure $v(\mathrm{~d} x)$.

\subsection{Clark-Ocone and Feynman-Kac formulae}

Let us consider the simple case of a BSDE where $f=0$, and the terminal random variable $\xi$ is a function of $X_{T}$, that is,

$$
-\mathrm{d} Y_{t}=-\sum_{i=1}^{\infty} Z_{t}^{(i)} \mathrm{d} H_{t}^{(i)}, \quad Y_{T}=g\left(X_{T}\right)
$$

or equivalently

$$
Y_{t}=g\left(X_{T}\right)-\sum_{i=1}^{\infty} \int_{t}^{T} Z_{s}^{(i)} \mathrm{d} H_{s}^{(i)}
$$


where $\mathrm{E}\left(g\left(X_{T}\right)^{2}\right)<\infty$. Let $\theta=\theta(t, x)$ be the solution of the following PDIE with terminal value $g$ :

$$
\begin{gathered}
\frac{\partial \theta}{\partial t}(t, x)+\int_{\mathbb{R}}\left(\theta(t, x+y)-\theta(t, x)-\frac{\partial \theta}{\partial x}(t, x) y\right) v(\mathrm{~d} y)+a^{\prime} \frac{\partial \theta}{\partial x}(t, x)=0, \\
\theta(T, x)=g(x),
\end{gathered}
$$

where $a^{\prime}=a+\int\{|y| \geqslant 1\} y v(\mathrm{~d} y)$. Set

$$
\theta^{(1)}(t, x, y)=\theta(t, x+y)-\theta(t, x)-\frac{\partial \theta}{\partial x}(t, x) y .
$$

The following result is a version of the Clark-Ocone formula for functions of a Lévy process. Again the proof can be found in the Appendix.

Proposition 3. Suppose that $\theta$ is a $C^{1,2}$ function such that $\partial \theta / \partial x$ and $\partial^{2} \theta / \partial x^{2}$ are bounded by a polynomial function of $x$, uniformly in $t$. Then the unique adapted solution of (3) is given by

$$
\begin{aligned}
Y_{t} & =\theta\left(t, X_{t}\right), \\
Z_{t}^{(1)} & =\int_{\mathbb{R}} \theta^{(1)}\left(t, X_{t-}, y\right) p_{1}(y) v(\mathrm{~d} y)+\frac{\partial \theta}{\partial x}\left(t, X_{t-}\right)\left(\int_{\mathbb{R}} y^{2} v(\mathrm{~d} y)\right)^{1 / 2}, \\
Z_{t}^{(i)} & =\int_{\mathbb{R}} \theta^{(1)}\left(t, X_{t-}, y\right) p_{i}(y) v(\mathrm{~d} y), \quad i \geqslant 2,
\end{aligned}
$$

where $\theta=\theta(t, x)$ is the solution of the PDIE (4) and $\theta^{(1)}(t, x, y)$ is given by (5).

Now by taking expectations we obtain that the solution $\theta(t, x)$ to our PDIE (4) equation has the stochastic representation

$$
\theta(t, x)=\mathrm{E}\left[g\left(X_{T}\right) \mid X_{t}=x\right] .
$$

This is an extension of the classical Feynman-Kac formula.

If $\int_{\mathbb{R}}|y| v(\mathrm{~d} y)<\infty$, and we take $a=\int_{\{|y|<1\}} y v(\mathrm{~d} y)$, then (4) reduces to

$$
\begin{gathered}
\frac{\partial \theta}{\partial t}(t, x)+\int_{\mathbb{R}}(\theta(t, x+y)-\theta(t, x)) v(\mathrm{~d} y)=0, \\
\theta(T, x)=g(x),
\end{gathered}
$$

and, taking into account that $p_{1}(y)=y\left(\int_{\mathbb{R}} y^{2} v(\mathrm{~d} y)\right)^{-1 / 2}$ in Proposition 3, we have

$$
Z_{t}^{(1)}=\int_{\mathbb{R}}\left[\theta\left(t, X_{t-}+y\right)-\theta\left(t, X_{t-}\right)\right] p_{1}(y) v(\mathrm{~d} y) .
$$

Example. Consider the very special case where we have a compensated Poisson process $X_{t}=N_{t}-\lambda t$. Then 
Backward stochastic differential equations and Feynman-Kac formula

$$
\begin{aligned}
& H_{t}^{(1)}=\frac{1}{\sqrt{\lambda}}\left(N_{t}-\lambda t\right)=\frac{X_{t}}{\sqrt{\lambda}}, \\
& H_{t}^{(i)}=0, \quad i=2,3, \ldots .
\end{aligned}
$$

Note that $p_{1}(x)=x / \sqrt{\lambda}$ and $p_{i}(x)=0, i=2,3, \ldots$. Moreover, the PDIE (4) reduces to

$$
\begin{gathered}
\left(\theta(t, x+1)-\theta(t, x)-\lambda \frac{\partial \theta}{\partial x}(t, x)+\frac{\partial \theta}{\partial t}(t, x)=0,\right. \\
\theta(T, x)=g(x) .
\end{gathered}
$$

The Clark-Ocone formula is now given by

$$
g\left(X_{T}\right)=\mathrm{E}\left[g\left(X_{T}\right)\right]+\int_{t}^{T} \theta\left(s, X_{s-}+1\right)-\theta\left(s, X_{s-}\right) \mathrm{d} X_{s} .
$$

\subsection{Nonlinear Clark-Haussman-Ocone and Feynman-Kac formulae}

Let us consider the BSDE

$$
-\mathrm{d} Y_{t}=f\left(t, Y_{t}, Z_{t}\right) \mathrm{d} t-\sum_{i=1}^{\infty} Z_{t}^{(i)} \mathrm{d} H_{t}^{(i)}, \quad Y_{T}=g\left(X_{T}\right)
$$

or equivalently,

$$
Y_{t}=g\left(X_{T}\right)+\int_{t}^{T} f\left(s, Y_{s-}, Z_{s}\right) \mathrm{d} s-\sum_{i=1}^{\infty} \int_{t}^{T} Z_{s}^{(i)} \mathrm{d} H_{s}^{(i)} .
$$

Suppose that $\theta=\theta(t, x)$ satisfies the following PDIE:

$$
\begin{gathered}
\frac{\partial \theta}{\partial t}(t, x)+\int_{\mathbb{R}} \theta^{(1)}(t, x, y) v(\mathrm{~d} y)+a^{\prime} \frac{\partial \theta}{\partial x}(t, x)+f\left(t, \theta(t, x),\left\{\theta^{(i)}(t, x)\right\}_{i=1}^{\infty}\right)=0, \\
\theta(T, x)=g(x) .
\end{gathered}
$$

where, as in the previous section, we define $\theta^{(1)}(t, x, y)$ by (5),

$$
\theta^{(1)}(t, x)=\int_{\mathbb{R}} \theta^{(1)}(t, x, y) p_{1}(y) v(\mathrm{~d} y)+\frac{\partial \theta}{\partial x}(t, x)\left(\int_{\mathbb{R}} y^{2} v(\mathrm{~d} y)\right)^{1 / 2},
$$

and, for $i \geqslant 2$,

$$
\theta^{(i)}(t, x)=\int_{\mathbb{R}} \theta^{(1)}(t, x, y) p_{i}(y) v(\mathrm{~d} y)
$$

Proposition 4. Suppose that $\theta$ is a $C^{1,2}$ function such that $\partial \theta / \partial x$ and $\partial^{2} \theta / \partial x^{2}$ are bounded by a polynomial function of $x$, uniformly in $t$. Then the (unique) adapted solution of (6) is given by 


$$
\begin{aligned}
Y_{t} & =\theta\left(t, X_{t}\right), \\
Z_{t}^{(1)} & =\int_{\mathbb{R}} \theta^{(1)}\left(t, X_{t-}, y\right) p_{1}(y) v(\mathrm{~d} y)+\frac{\partial \theta}{\partial x}\left(t, X_{t-}\right)\left(\int_{\mathbb{R}} y^{2} v(\mathrm{~d} y)\right)^{1 / 2}, \\
Z_{t}^{(i)} & =\int_{\mathbb{R}} \theta^{(1)}\left(t, X_{t-}, y\right) p_{i}(y) v(\mathrm{~d} y), \quad i \geqslant 2,
\end{aligned}
$$

where $\theta=\theta(t, x)$ is the solution of the PDIE (7) and $\theta^{(1)}(t, x, y)$ is given by (5).

Notice that, taking expectations, we get

$$
\theta(t, x)=\mathrm{E}\left[g\left(X_{T}\right) \mid X_{t}=x\right]+\mathrm{E}\left[\int_{t}^{T} f\left(s, \theta\left(s, X_{s-}\right),\left\{\theta^{(i)}\left(s, X_{s-}\right)\right\}_{i=1}^{\infty}\right) \mathrm{d} s \mid X_{t}=x\right] .
$$

Example. Consider again the very special case where we have a Poisson process $N_{t}$ with $\mathrm{E}\left[N_{t}\right]=\lambda t$. Set $X_{t}=N_{t}-\lambda t$. Then the PDIE (7) reduces to

$$
\begin{gathered}
(\theta(t, x+1)-\theta(t, x))-\lambda \frac{\partial \theta}{\partial x}(t, x)+\frac{\partial \theta}{\partial t}(t, x)+f(t, \theta(t, x), \theta(t, x+1)-\theta(t, x))=0, \\
\theta(T, x)=g(x),
\end{gathered}
$$

and we derive the nonlinear Feynman-Kac formula,

$$
\theta(t, x)=\mathrm{E}\left[g\left(X_{T}\right) \mid X_{t}=x\right]+\mathrm{E}\left[\int_{t}^{T} f\left(s, \theta\left(s, X_{s-}\right), \theta\left(s, X_{s-}+1\right)-\theta\left(s, X_{s-}\right)\right) \mathrm{d} s \mid X_{t}=x\right] .
$$

\subsection{Option pricing}

Assume a market consisting of one riskless asset (the bond) with price process given by $B_{t}=\mathrm{e}^{r t}$, where $r$ is compound interest rate, and one risky asset (the stock), with price process

$$
S_{t}=S_{0} \exp \left(X_{t}\right)
$$

where $X_{t}$ is a Lévy process. Denote by $P(\mathrm{~d} x)$ the probability measure of $X_{1}$.

In the last two decades several particular choices for non-Brownian Lévy processes have been proposed. Madan and Seneta (1990) have proposed a Lévy process with variance gamma distributed increments. We mention also the hyperbolic model proposed by Eberlein and Keller (1995). In the same year Barndorff-Nielsen (1995) proposed the normal inverse Gaussian Lévy process. Recently, Carr et al. (2000) introduced the CMGY model, which generalizes the variance Gamma model. Finally, we mention the Meixner model (see Grigelionis 1999; Schoutens 2001). All these models give a much better fit to the data and lead to an improvement with respect to the Black-Scholes model. 
We recall the density $f$, the cumulant generating function $K$, the drift $a$, and the Lévy measure $v$, for the Meixner process $\left\{M_{t}, t \geqslant 0\right\}$, for which we will illustrate the method:

$$
\begin{aligned}
\frac{P_{\text {Meixner }}(\mathrm{d} x)}{\mathrm{d} x}=f_{\text {Meixner }}(x ; \alpha, \beta, \delta, \mu) & =\frac{(2 \cos (\beta / 2))^{2 \delta} \exp (\beta(x-\mu) / \alpha)(|\Gamma(\delta+\mathrm{i}(x-\mu) / \alpha)|)^{2}}{\pi \alpha \Gamma(2 \delta)}, \\
K_{\text {Meixner }}(\theta ; \alpha, \beta, \delta, \mu) & =\mu \theta+2 \delta\left(\log \cos \frac{\beta}{2}-\log \cos \frac{\alpha \theta+\beta}{2}\right) \\
a_{\text {Meixner }}(\alpha, \beta, \delta, \mu) & =\mu+\alpha \delta \tan \frac{\beta}{2}-2 \delta \int_{1}^{\infty} \frac{\sinh (\beta x / \alpha)}{\sinh (\pi x / \alpha)} \mathrm{d} x \\
v_{\text {Meixner }}(\mathrm{d} x ; \alpha, \beta, \delta, \mu) & =\frac{\delta \exp (\beta x / \alpha)}{x \sinh (\pi x / \alpha)} \mathrm{d} x
\end{aligned}
$$

where $\alpha>0,-\pi<\beta<\pi, \mu \in \mathbb{R}$ and $\delta>0$.

From the form of the cumulant generating function one easily deduces that the density at any time $t$ can be calculated by multiplying the parameters $\delta$ and $\mu$ by $t$ for both cases.

Given our market model, let $G\left(S_{T}\right)=F\left(X_{T}\right)$ denote the pay-off of the derivative at its time of expiry $T$. In case of a European call with strike price $K$, we have $G\left(S_{T}\right)=$ $\left(S_{T}-K\right)^{+}$or, equivalently, $F\left(X_{T}\right)=\left(S_{0} \exp \left(X_{T}\right)-K\right)^{+}$. According to the fundamental theorem of asset pricing (see Delbaen and Schachermayer 1994), the arbitrage free price $V_{t}$ of the derivative at time $t \in[0, T]$ is given by

$$
V_{t}=\mathrm{E}_{Q}\left[\mathrm{e}^{-r(T-t)} G\left(S_{T}\right) \mid \mathscr{F}_{t}\right],
$$

where the expectation is taken with respect to an equivalent martingale measure $Q(\mathrm{~d} x)$ and $\mathscr{F}=\left\{\mathscr{F}_{t}, 0 \leqslant t \leqslant T\right\}$ is the natural filtration of $X=\left\{X_{t}, 0 \leqslant t \leqslant T\right\}$. An equivalent martingale measure is a probability measure which is equivalent (it has the same null sets) to the given (historical) probability measure and under which the discounted process $\left\{\mathrm{e}^{-r t} S_{t}\right\}$ is a martingale. Unfortunately for most models, in particular the more realistic ones, the class of equivalent measures is rather large and often covers the full no-arbitrage interval. In this perspective the Black-Scholes model, where there is an unique equivalent martingale measure, is very exceptional. Models with more than one equivalent measure are called 'incomplete'.

Our Lévy model is such an incomplete model. Following Gerber and Shiu (1994; 1996), we can, by using the so-called Esscher transform, easily find at least one equivalent martingale measure, which we will use below for the valuation of derivative securities. The choice of the Esscher measure may be justified by a utility maximizing argument (see Gerber and Shiu 1996).

Let $K$ be the cumulant generating function of $X$ under the measure $P(\mathrm{~d} x)$, and let $\theta$ be the solution of $K(\theta+1)-K(\theta)=r$. Then we define the risk-neutral measure $Q(\mathrm{~d} x)$ as the probability measure with the Radon-Nykodym derivative with respect to $P(\mathrm{~d} x)$ given by $Q(\mathrm{~d} x) / P(\mathrm{~d} x)=\exp (\theta x-K(\theta))$.

For our Meixner example, the parameters for the Esscher transforms are easily found; explicit values for $\theta$ can be found in Schoutens (2001) or Grigelionis (1999). In the 
Meixner case one only has to shift $\beta$ to $\beta+\alpha \theta$ to obtain the density under the measure $Q(\mathrm{~d} x)$. This means that under the risk-neutral measure $Q(\mathrm{~d} x)$ our process $M_{t}$ is again a Meixner process. In all such cases where the underlying process is a Lévy process in the risk-neutral world and the price $V_{t}=V\left(t, M_{t}\right)$ at time $t$ of a given derivative satisfies some regularity conditions (i.e. $\left.V(t, x) \in C^{(1,2)}\right)$, the function $V(t, x)$ can also be obtained by solving a PDIE with a boundary condition:

$$
\begin{aligned}
r V(t, x) & =a \frac{\partial}{\partial x} V(t, x)+\frac{\partial}{\partial t} V(t, x)+\int_{-\infty}^{+\infty}\left(V(t, x+y)-V(t, x)-y \frac{\partial}{\partial x} V(t, x)\right) v^{Q}(\mathrm{~d} y), \\
V(T, x) & =F(x),
\end{aligned}
$$

where $v^{Q}(\mathrm{~d} y)$ is the Lévy measure of the risk-neutral distribution $Q(\mathrm{~d} x)$. This PDIE is the analogue of the famous Black-Scholes partial differential equation and follows from the above Feynman-Kac formula for Lévy processes. In the Meixner case, it is clear that

$$
v^{Q}(\mathrm{~d} x)=d \frac{\exp ((a \theta+b) x / a)}{x \sinh (\pi x / a)} \mathrm{d} x
$$

and $a=a_{\text {Meixner }}(\alpha, \alpha \theta+\beta, \delta, \mu)$.

\section{Appendix: Proofs of the Results}

Proof of Theorem 1. We define a mapping $\Phi$ from $\mathscr{H}_{T}^{2}$ into itself such that $(Y, Z) \in \mathscr{H}_{T}^{2}$ is a solution of the BSDE if and only if it is a fixed point of $\Phi$. Given $(U, V) \in \mathscr{H}_{T}^{2}$, we define $(Y, Z)=\Phi(U, V)$ as follows:

$$
Y_{t}=\mathrm{E}\left[\xi+\int_{t}^{T} f\left(s, U_{s-}, V_{s}\right) \mathrm{d} s \mid \mathscr{F}_{t}\right], \quad 0 \leqslant t \leqslant T
$$

and $\left\{Z_{t}, 0 \leqslant t \leqslant T\right\}$ is given by the martingale representation of Nualart and Schoutens (2000) applied to the square-integrable random variable

$$
\xi+\int_{0}^{T} f\left(s, U_{s-}, V_{s}\right) \mathrm{d} s
$$

that is,

$$
\xi+\int_{0}^{T} f\left(s, U_{s-}, V_{s}\right) \mathrm{d} s=\mathrm{E}\left[\xi+\int_{0}^{T} f\left(s, U_{s-}, V_{s}\right) \mathrm{d} s\right]+\sum_{i=1}^{\infty} \int_{0}^{T} Z_{s}^{(i)} \mathrm{d} H_{s}^{(i)} .
$$

Taking the conditional expectation with respect to $\mathscr{T}_{t}$ in the last identity yields

$$
Y_{t}+\int_{0}^{t} f\left(s, U_{s-}, V_{s}\right) \mathrm{d} s=Y_{0}+\sum_{i=1}^{\infty} \int_{0}^{t} Z_{s}^{(i)} \mathrm{d} H_{s}^{(i)},
$$

from which we deduce that 


$$
Y_{t}=\xi+\int_{t}^{T} f\left(s, U_{s-}, V_{s}\right) \mathrm{d} s-\sum_{i=1}^{\infty} \int_{t}^{T} Z_{s}^{(i)} \mathrm{d} H_{s}^{(i)}
$$

and we have shown that $(Y, Z) \in \mathscr{H}_{T}^{2}$ solves our BSDE if and only if it is a fixed point of $\Phi$.

Next we prove that $\Phi$ is a strict contraction on $\mathscr{H}_{T}^{2}$ equipped with the norm

$$
\|(Y, Z)\|_{\beta}=\left(\mathrm{E}\left[\int_{0}^{T} \mathrm{e}^{\beta s}\left(\left|Y_{s-}\right|^{2}+\sum_{i=1}^{\infty}\left|Z_{s}^{(i)}\right|^{2}\right) \mathrm{d} s\right]\right)^{1 / 2},
$$

for a suitable $\beta>0$. Let $(U, V)$ and $\left(U^{\prime}, V^{\prime}\right)$ be two elements of $\mathscr{H}_{T}^{2}$ and set $\Phi(U, V)=(Y, Z)$ and $\Phi\left(U^{\prime}, V^{\prime}\right)=\left(Y^{\prime}, Z^{\prime}\right)$. Denote $(\bar{U}, \bar{V})=\left(U-U^{\prime}, V-V^{\prime}\right)$ and $(\bar{Y}, \bar{Z})=\left(Y-Y^{\prime}, Z-Z^{\prime}\right)$.

Applying Itô's formula to $\mathrm{e}^{\beta s}\left(Y_{s}-Y_{s}^{\prime}\right)^{2}$, from $s=t$ to $s=T$, it follows that

$$
\begin{aligned}
\mathrm{e}^{\beta t}\left(Y_{t}-Y_{t}^{\prime}\right)^{2}= & -\beta \int_{t}^{T} \mathrm{e}^{\beta s}\left(Y_{s-}-Y_{s-}^{\prime}\right)^{2} \mathrm{~d} s-2 \int_{t}^{T} \mathrm{e}^{\beta s}\left(Y_{s-}-Y_{s-}^{\prime}\right) \mathrm{d}\left(Y_{s}-Y_{s}^{\prime}\right) \\
& -\int_{t}^{T} \mathrm{e}^{\beta s} \mathrm{~d}\left[Y-Y^{\prime}, Y-Y^{\prime}\right]_{s} .
\end{aligned}
$$

We have

$$
\begin{aligned}
-\mathrm{d}\left(Y_{t}-Y_{t}^{\prime}\right) & =\left(f\left(t, U_{t-}, V_{t}\right)-f\left(t, U_{t-}^{\prime}, V_{t}^{\prime}\right)\right) \mathrm{d} t-\sum_{i=1}^{\infty}\left(Z_{t}^{(i)}-Z_{t}^{\prime(i)}\right) \mathrm{d} H_{t}^{(i)}, \\
\mathrm{d}\left[Y-Y^{\prime}, Y-Y^{\prime}\right]_{t} & =\sum_{i=1}^{\infty} \sum_{j=1}^{\infty}\left(Z_{t}^{(i)}-Z_{t}^{\prime(i)}\right)\left(Z_{t}^{(j)}-Z_{t}^{(j)}\right) \mathrm{d}\left[H^{(i)}, H^{(j)}\right]_{t}, \\
\left\langle H^{(i)}, H^{(j)}\right\rangle_{t} & =\delta_{i j} t .
\end{aligned}
$$

Hence, taking expectations in (11), we have

$$
\begin{gathered}
\mathrm{E}\left[\mathrm{e}^{\beta t}\left(Y_{t}-Y_{t}^{\prime}\right)^{2}\right]+\sum_{i=1}^{\infty} \mathrm{E}\left[\int_{t}^{T} \mathrm{e}^{\beta s}\left(Z_{s}^{(i)}-Z_{s}^{\prime(i)}\right)^{2} \mathrm{~d} s\right] \\
=-\beta \mathrm{E}\left[\int_{t}^{T} \mathrm{e}^{\beta s}\left(Y_{s-}-Y_{s-}^{\prime}\right)^{2} \mathrm{~d} s\right]+2 \mathrm{E}\left[\int_{t}^{T} \mathrm{e}^{\beta s}\left(Y_{s-}-Y_{s-}^{\prime}\right)\left(f\left(s, U_{s-}, V_{s}\right)-f\left(s, U_{s-}^{\prime}, V_{s}^{\prime}\right)\right) \mathrm{d} s\right] .
\end{gathered}
$$

Using the fact that $f$ is Lipschitz with constant $C$ yields

$$
\begin{gathered}
\mathrm{E}\left[\mathrm{e}^{\beta t}\left(Y_{t}-Y_{t}^{\prime}\right)^{2}\right]+\sum_{i=1}^{\infty} \mathrm{E}\left[\int_{t}^{T} \mathrm{e}^{\beta s}\left(Z_{s}^{(i)}-Z_{s}^{\prime(i)}\right)^{2} \mathrm{~d} s\right] \leqslant-\beta \mathrm{E}\left[\int_{t}^{T} \mathrm{e}^{\beta s}\left(Y_{s-}-Y_{s-}^{\prime}\right)^{2} \mathrm{~d} s\right] \\
+2 C \mathrm{E}\left[\int_{t}^{T} \mathrm{e}^{\beta s}\left|Y_{s-}-Y_{s-}^{\prime}\right|\left(\left|U_{s-}-U_{s-}^{\prime}\right|+\sqrt{\sum_{i=1}^{\infty}\left|V_{s}^{(i)}-V_{s}^{(i)}\right|^{2}}\right) \mathrm{d} s\right] .
\end{gathered}
$$


If we now use the fact that for every $c>0$ and $a, b \in \mathbb{R}$ we have that $2 a b \leqslant c a^{2}+b^{2} / c$ and $(a+b)^{2} \leqslant 2 a^{2}+2 b^{2}$, we obtain

$$
\begin{gathered}
\mathrm{E}\left[\mathrm{e}^{\beta t}\left|Y_{t}-Y_{t}^{\prime}\right|^{2}\right]+\sum_{i=1}^{\infty} \mathrm{E}\left[\int_{t}^{T} \mathrm{e}^{\beta s}\left(Z_{s}^{(i)}-Z_{s}^{\prime(i)}\right)^{2} \mathrm{~d} s\right] \\
\leqslant\left(4 C^{2}-\beta\right) \mathrm{E}\left[\int_{t}^{T} \mathrm{e}^{\beta s}\left|Y_{s}-Y_{s}^{\prime}\right|^{2} \mathrm{~d} s\right]+\frac{1}{2} \mathrm{E}\left[\int_{t}^{T} \mathrm{e}^{\beta s}\left(\left|U_{s-}-U_{s-}^{\prime}\right|^{2}+\sum_{i=1}^{\infty}\left|V_{s}^{(i)}-V_{s}^{\prime(i)}\right|^{2}\right) \mathrm{d} s\right] .
\end{gathered}
$$

Taking $\beta=4 C^{2}+1$, and noting that $\mathrm{e}^{\beta t} \mathrm{E}\left[\left(Y_{t}-Y_{t}^{\prime}\right)^{2}\right] \geqslant 0$, we finally obtain

$$
\begin{gathered}
\mathrm{E}\left[\int_{t}^{T} \mathrm{e}^{\beta s}\left|Y_{s}-Y_{s}^{\prime}\right|^{2} \mathrm{~d} s\right]+\sum_{i=1}^{\infty} \mathrm{E}\left[\int_{t}^{T} \mathrm{e}^{\beta s}\left(Z_{s}^{(i)}-Z_{s}^{\prime(i)}\right)^{2} \mathrm{~d} s\right] \\
\leqslant \frac{1}{2} \mathrm{E}\left[\int_{t}^{T} \mathrm{e}^{\beta s}\left(\left|U_{s-}-U_{s-}^{\prime}\right|^{2}+\sum_{i=1}^{\infty}\left|V_{s}^{(i)}-V_{s}^{\prime(i)}\right|^{2}\right) \mathrm{d} s\right],
\end{gathered}
$$

that is,

$$
\|(Y, Z)\|_{\beta}^{2} \leqslant \frac{1}{2}\|(U, V)\|_{\beta}^{2}
$$

from which it follows that $\Phi$ is a strict contraction on $\mathscr{H}_{T}^{2}$ equipped with the norm $\|\cdot\|_{\beta}$ if $\beta=4 C^{2}+1$. Then $\Phi$ has a unique fixed point and the theorem is proved.

Proof of Theorem 2. Applying Itô's formula to $\left(Y_{s}-Y_{s}^{\prime}\right)^{2}$, from $s=t$ to $s=T$, it follows that

$$
\left(Y_{T}-Y_{T}^{\prime}\right)^{2}-\left(Y_{t}-Y_{t}^{\prime}\right)^{2}=2 \int_{t}^{T}\left(Y_{s-}-Y_{s-}^{\prime}\right) \mathrm{d}\left(Y_{s}-Y_{s}^{\prime}\right)+\int_{t}^{T} \mathrm{~d}\left[Y-Y^{\prime}, Y-Y^{\prime}\right]_{s} .
$$

Taking expectations and using the relations

$$
\begin{aligned}
-\mathrm{d}\left(Y_{t}-Y_{t}^{\prime}\right) & =f\left(t, Y_{t-}, Z_{t}\right)-f^{\prime}\left(t, Y_{t-}^{\prime}, Z_{t}^{\prime}\right) \mathrm{d} t-\sum_{i=1}^{\infty}\left(Z_{t}^{(i)}-{Z^{\prime}}_{t}^{(i)}\right) \mathrm{d} H_{t}^{(i)} \\
\mathrm{d}\left[Y-Y^{\prime}, Y-Y^{\prime}\right]_{t} & =\sum_{i=1}^{\infty} \sum_{j=1}^{\infty}\left(Z_{t}^{(i)}-Z^{\prime(i)}\right)\left(Z_{t}^{(j)}-Z_{t}^{\prime(j)}\right) \mathrm{d}\left[H^{(i)}, H^{(j)}\right]_{t} \\
\left\langle H^{(i)}, H^{(j)}\right\rangle_{t} & =\delta_{i j} t
\end{aligned}
$$

we have 


$$
\begin{aligned}
\mathrm{E}\left[\left(Y_{t}-Y_{t}^{\prime}\right)^{2}\right]+\sum_{i=1}^{\infty} & \mathrm{E}\left[\int_{t}^{T}\left|Z_{s}^{(i)}-Z_{s}^{\prime(i)}\right|^{2} \mathrm{~d} s\right] \\
& =\mathrm{E}\left[\left(\xi-\xi^{\prime}\right)^{2}\right]+2 \mathrm{E}\left[\int_{t}^{T}\left(Y_{s-}-Y_{s-}^{\prime}\right)\left(f\left(s, Y_{s-}, Z_{s}\right)-f^{\prime}\left(s, Y_{s-}^{\prime}, Z_{s}^{\prime}\right)\right) \mathrm{d} s\right] .
\end{aligned}
$$

Using the Lipschitz property of $f^{\prime}$ and computations similar to those of the proof of Theorem 1 , we obtain

$$
\begin{aligned}
\mathrm{E}\left[\left|Y_{t}-Y_{t}^{\prime}\right|^{2}\right]+\frac{1}{2} \mathrm{E}\left[\int_{t}^{T} \sum_{i=1}^{\infty}\left|Z_{s}^{(i)}-Z_{s}^{\prime(i)}\right|^{2} \mathrm{~d} s\right] \\
\leqslant \mathrm{E}\left[\left|\xi-\xi^{\prime}\right|^{2}\right]+\left(1+2 C^{\prime}+2 C^{\prime 2}\right) \mathrm{E}\left[\int_{t}^{T}\left|Y_{s-}-Y_{s-}^{\prime}\right|^{2} \mathrm{~d} s\right] \\
+\mathrm{E}\left[\int_{t}^{T} \mid f\left(s, Y_{s-}, Z_{s}\right)-f^{\prime}\left(s, Y_{s-},\left.Z_{s}\right|^{2} \mathrm{~d} s\right] .\right.
\end{aligned}
$$

Then, by Gronwall's inequality, the result follows.

Lemma 5. Let $h: \Omega \times[0, T] \times \mathbb{R} \rightarrow \mathbb{R}$ be a random function measurable with respect to $\mathscr{P} \otimes B_{\mathbb{R}}$ such that

$$
|h(s, y)| \leqslant a_{s}\left(y^{2} \wedge|y|\right) \text { a.s. }
$$

where $\left\{a_{s}, 0 \leqslant s \leqslant T\right\}$ is a non-negative predictable process such that $\mathrm{E}\left[\int_{0}^{T} a_{s}^{2} \mathrm{~d} s\right]<\infty$. Then, for each $t \in[0, T]$, we have

$$
\sum_{t<s \leqslant T} h\left(s, \Delta X_{s}\right)=\sum_{i=1}^{\infty} \int_{t}^{T}\left\langle h(s, \cdot), p_{i}\right\rangle_{L^{2}(v)} \mathrm{d} H_{s}^{(i)}+\int_{t}^{T} \int_{\mathbb{R}} h(s, y) v(\mathrm{~d} y) \mathrm{d} s .
$$

Proof. Because (12) implies that $\mathrm{E}\left[\int_{0}^{t} \int_{\mathbb{R}}|h(s, y)|^{2} v(\mathrm{~d} y) \mathrm{d} s\right]<\infty$, we have that

$$
M_{t}=\sum_{0<s \leqslant t} h\left(s, \Delta X_{s}\right)-\int_{0}^{t} \int_{\mathbb{R}} h(s, y) v(\mathrm{~d} y) \mathrm{d} s
$$

is a square-integrable martingale. By the predictable representation theorem, there exists a process $\phi$ in the space $M_{T}^{2}\left(l^{2}\right)$ such that

$$
M_{t}=\sum_{i=1}^{\infty} \int_{0}^{t} \phi_{s}^{(i)} \mathrm{d} H_{s}^{(i)}
$$

Taking into account that $\left\langle H^{(i)}, H^{(j)}\right\rangle_{t}=t \delta_{j i}$, we have

$$
\left\langle M, H^{(i)}\right\rangle_{t}=\int_{0}^{t} \phi_{s}^{(i)} \mathrm{d} s .
$$


On the other hand, using the fact that $\Delta M_{s} \Delta H_{s}^{(i)}=h\left(s, \Delta X_{s}\right) p_{i}\left(\Delta X_{s}\right)$, we obtain

$$
\left\langle M, H^{(i)}\right\rangle_{t}=\int_{0}^{t} \int_{\mathbb{R}} h(s, y) p_{i}(y) v(\mathrm{~d} y) \mathrm{d} s .
$$

Consequently, (13) and (14) imply that

$$
\phi_{s}^{(i)}=\int_{\mathbb{R}} h(s, y) p_{i}(y) v(\mathrm{~d} y),
$$

and the result follows.

Proof of Proposition 3. Under the hypotheses of Proposition 3 the function $\theta^{(1)}(t, x, y)$ given by (5) satisfies the hypotheses in Lemma 5 imposed on $h$ due to the mean value theorem, when we take $x=X_{t-}$.

Apply Itô's lemma to $\theta\left(s, X_{s}\right)$, from $s=t$ to $s=T$ :

$$
\begin{aligned}
\theta\left(T, X_{T}\right)-\theta\left(t, X_{t}\right)= & \int_{t}^{T} \frac{\partial \theta}{\partial t}\left(s, X_{s-}\right) \mathrm{d} s+\int_{t}^{T} \frac{\partial \theta}{\partial x}\left(s, X_{s-}\right) \mathrm{d} X_{s} \\
& +\sum_{t<s \leqslant T}\left[\theta\left(s, X_{s}\right)-\theta\left(s, X_{s-}\right)-\frac{\partial \theta}{\partial x}\left(s, X_{s-}\right) \Delta X_{s}\right] .
\end{aligned}
$$

If we apply Lemma 5 to

$$
h(s, y)=\theta\left(s, X_{s-}+y\right)-\theta\left(s, X_{s-}\right)-\frac{\partial \theta}{\partial x}\left(s, X_{s-}\right) y,
$$

we obtain

$$
\begin{aligned}
\sum_{t<s \leqslant T}\left[\theta\left(s, X_{s}\right)-\theta\left(s, X_{s-}\right)-\frac{\partial \theta}{\partial x}\left(s, X_{s-}\right) \Delta X_{s}\right]= & \sum_{i=1}^{\infty} \int_{t}^{T}\left(\int_{\mathbb{R}} \theta^{(1)}\left(s, X_{s-}, y\right) p_{i}(y) v(\mathrm{~d} y)\right) \mathrm{d} H_{s}^{(i)} \\
& +\int_{t}^{T} \int_{\mathbb{R}} \theta^{(1)}\left(s, X_{s-}, y\right) v(\mathrm{~d} y) \mathrm{d} s .
\end{aligned}
$$

Hence, substituting (16) into (15) yields

$$
\begin{aligned}
g\left(X_{T}\right)-\theta\left(t, X_{t}\right)= & \int_{t}^{T} \frac{\partial \theta}{\partial t}\left(s, X_{s-}\right) \mathrm{d} s+\int_{t}^{T} \frac{\partial \theta}{\partial x}\left(s, X_{s-}\right) \mathrm{d} X_{s} \\
& +\sum_{i=1}^{\infty} \int_{t}^{T}\left(\int_{\mathbb{R}} \theta^{(1)}\left(s, X_{s-}, y\right) p_{i}(y) v(\mathrm{~d} y)\right) \mathrm{d} H_{s}^{(i)} \\
& +\int_{t}^{T} \int_{\mathbb{R}} \theta^{(1)}\left(s, X_{s-}, y\right) v(\mathrm{~d} y) \mathrm{d} s
\end{aligned}
$$

Notice that 


$$
X_{t}=Y_{t}^{(1)}+t \mathrm{E}\left(X_{1}\right)=\left(\int_{\mathbb{R}} y^{2} v(\mathrm{~d} y)\right)^{1 / 2} H_{t}^{(1)}+t \mathrm{E}\left(X_{1}\right)
$$

and

$$
\mathrm{E}\left(X_{1}\right)=a+\int_{\{|y| \geqslant 1\}} y v(\mathrm{~d} y)
$$

We also have $Y_{0}=\mathrm{E}\left[Y_{0}\right]=\mathrm{E}\left[g\left(\mathrm{X}_{T}\right)\right]$, so we can rewrite (17) as

$$
\begin{aligned}
g\left(X_{T}\right)= & \mathrm{E}\left[g\left(X_{T}\right)\right]+\int_{t}^{T} \frac{\partial \theta}{\partial x}\left(s, X_{s-}\right)\left(\int_{\mathbb{R}} y^{2} v(\mathrm{~d} y)\right)^{1 / 2} \mathrm{~d} H_{s}^{(1)} \\
& +\sum_{i=1}^{\infty} \int_{t}^{T}\left(\int_{\mathbb{R}} \theta^{(1)}\left(s, X_{s-}, y\right) p_{i}(y) v(\mathrm{~d} y)\right) \mathrm{d} H_{s}^{(i)},
\end{aligned}
$$

which completes the proof of the proposition.

Proof of Proposition 4. Apply Itô's lemma to $\theta\left(s, X_{s}\right)$, from $s=t$ to $s=T$. By using Lemma 5, we obtain (17). Now, using (7), we obtain

$$
\begin{aligned}
g\left(X_{T}\right)-\theta\left(t, X_{t}\right)= & -\int_{t}^{T} f\left(s, \theta\left(s, X_{s-}\right),\left\{\theta^{(k)}\left(s, X_{s-}\right)\right\}_{k=1}^{\infty}\right) \mathrm{d} s \\
& +\int_{t}^{T}\left[\int_{\mathbb{R}} \theta^{(1)}\left(s, X_{s-}, y\right) p_{1}(y) v(\mathrm{~d} y)+\frac{\partial \theta}{\partial x}\left(s, X_{s-}\right)\left(\int_{\mathbb{R}} y^{2} v(\mathrm{~d} y)\right)^{1 / 2}\right] \mathrm{d} H_{s}^{(1)} \\
& +\sum_{i=2}^{\infty} \int_{t}^{T}\left[\int_{\mathbb{R}} \theta^{(1)}\left(s, X_{s-}, y\right) p_{i}(y) v(\mathrm{~d} y)\right] \mathrm{d} H_{s}^{(i)},
\end{aligned}
$$

which completes the proof of the Proposition.

\section{Acknowledgement}

The second named author is a postdoctoral fellow of the Fund for Scientific Research Flanders, Belgium (FWO - Vlaanderen). The authors would like to thank the referees for their useful suggestions for improvement of the manuscript.

\section{References}

Barndorff-Nielsen, O.E. (1995) Normal inverse Gaussian distributions and the modeling of stock returns. Research Report No. 300, Department of Theoretical Statistics, Aarhus University.

Bertoin, J. (1996) Lévy Processes, Cambridge Tracts in Math. 121. Cambridge: Cambridge University Press. 
Black, F. and Scholes, M. (1973) The pricing of options and corporate liabilities. J. Political Economy, 81, 637-654.

Bismut, J.M. (1973) Conjugate convex functions in optimal stochastic control. J. Math. Anal. Appl., 44, 384-404.

Carr, P., Geman, H., Madan, D.H. and Yor, M. (2000) The fine structure of asset returns: an empirical investigation. Preprint.

Delbaen, F. and Schachermayer, W. (1994) A general version of the fundamental theorem of asset pricing. Math. Ann., 300, 463-520.

Eberlein, E. and Keller, U. (1995) Hyperbolic distributions in finance. Bernoulli, 1, 281-299.

El Karoui, N. and Quenez, M.C. (1997) Non-linear pricing theory and backward stochastic differential equations. In W.J. Runggaldier (ed.), Financial Mathematics, Lecture Notes in Math. 1656, pp. 191-246. Berlin: Springer-Verlag.

Gerber, H.U. and Shiu, E.S.W (1994) Option pricing by Esscher-transforms. Trans. Soc. Actuaries, 46, 99-191.

Gerber, H.U. and Shiu, E.S.W (1996) Actuarial bridges to dynamic hedging and option pricing. Insurance Math. Econom., 18(3), 183-218.

Grigelionis, B. (1999) Processes of Meixner type. Lithuanian Math. J., 39(1), 33-41.

Jacod, J. (1979) Calcul Stochastique et Problèmes de Martingales, Lecture Notes in Math. 714. Berlin: Springer-Verlag.

Ma, J. and Yong, J. (1999) Forward-Backward Stochastic Differential Equations and Their Applications, Lecture Notes in Math. 1702. Berlin: Springer-Verlag.

Madan, D.B. and Seneta, E. (1990) The variance gamma (V.G.) model for share market returns. $J$. Business, 63, 511-524.

Nualart, D. and Schoutens W. (2000) Chaotic and predictable representations for Lévy processes. Stochastic Process. Appl., 109-122.

Ouknine, Y. (1998) Reflected backward stochastic differential equations with jumps. Stochastics Stochastics Rep., 65, 111-125.

Pardoux, E. (1998) Backward stochastic differential equations and viscosity solutions of systems of semilinear parabolic and elliptic PDEs of second order. In L. Decreusefond et al. (eds), Stochastic Analysis and Related Topics VI. Proceedings of the Sixth Oslo-Silivri Workshop, Geilo, 1996, Progr. Probab. 42, pp. 79-127. Boston: Birkhäuser.

Pardoux, E. and Peng, S. (1990) Adapted solution of a backward stochastic differential equation. Systems Control Lett., 14, 55-61.

Sato, K. (2000) Lévy Processes and Infinitely Divisible Distributions, Cambridge Stud. Adv. Math. 68. Cambridge: Cambridge University Press.

Schoutens, W. (2001) The Meixner process in finance. EURANDOM-Report 2001-02, Eurandom, Eindhoven, Netherlands.

Situ, R. (1997) On solutions of backward stochastic differential equations with jumps and applications. Stochastic Process. Appl., 66, 209-236.

Received October 2000 and revised March 2001 